\title{
EFEITO DO ESTRESSE HÍDRICO NA GERMINAÇÃO E CRESCIMENTO INICIAL DE TRÊS ESPÉCIES DE LEGUMINOSAS
}

\author{
Eliane Romanato Santarém ${ }^{1}$ \\ Jarcilene S. Almeida-Cortez ${ }^{1}$ \\ Tânia Sales da Silveira ${ }^{1}$ \\ Alfredo Gui Ferreira ${ }^{2}$
}

Recebido em 6/3/95. Aceito em 29/5/96.

\begin{abstract}
RESUMO - (Efeito do estresse hídrico na germinação e crescimento inicial de três espécies de Leguminosas). Sementes de Senna macranthera, S. multijuga e Mimosa bimucronata foram submetidas a diferentes potenciais osmóticos simulados com PEG $6000(0 ;-0,15 ;-0,49 ;-1,03$ $\mathrm{MPa}$ ) com o objetivo de avaliar o efeito do estresse hídrico na germinação e crescimento inicial de plântulas. Os potenciais osmóticos de $-0,15$ e - $-0,49 \mathrm{MPa}$ causaram um retardo de 12,24 e 48 horas no início da germinação de $M$. bimucronata, $S$. multijuga e $S$. macranthera, respectivamente. Em todas as espécies, a germinabilidade final das sementes submetidas ao $\Psi=-0,49 \mathrm{MPa}$ foi reduzida e nenhuma semente germinou em solução de $\Psi=-1,03 \mathrm{MPa}$. Estes resultados indicam um limite de tolerância entre os potenciais $-0,49 \mathrm{e}-1,03 \mathrm{MPa}$. Plântulas de $S$. macranthera mostraram-se mais sensíveis ao estresse hídrico, reduzindo comprimentos de parte aérea e radícula à medida que os potenciais osmóticos tornavam-se mais negativos. Plântulas de $S$. multijuga e $M$. bimucronata apresentaram um aumento no comprimento médio da radícula em solução de $\Psi=-0,15 \mathrm{MPa}$. Houve redução do peso seco das plântulas a partir do $\Psi=-0,49 \mathrm{MPa}$ para as 3 espécies testadas.
\end{abstract}

Palavras-chaves: estresse hídrico, PEG 6000, germinação, Leguminosae, plântulas.

ABSTRACT - (Water stress effect on germination and initial growth of three Leguminosae
species). Seeds of Senna macranthera, $S$. multijuga e Mimosa bimucronata were submitted to
different osmotic potentials using PEG $6000(0 ;-0.15 ;-0.49$ and $-1.03 \mathrm{MPa})$. The objective of this
study was to evaluate the effect of osmotic stress on germinability and initial growth of seedlings.
Water stress of -0.15 and $-0.49 \mathrm{MPa}$ increased the time required for germination in 12,24 and 48
hours for M.bimucronata, $S$. multijuga and $S$. macranthera, respectively. All species showed a

Mestre em Ciências Biológicas - Botânica. Universidade Federal do Rio Grande do Sul (UFRGS)

Professor Titular do Depart. de Botânica, Instituto de Biociências, Universidade Federal do Rio Grande
do Sul (UFRGS), Porto Alegre - RS, CEP 90040-060, Brasil 
reduced germinability in the $\Psi=-1.03 \mathrm{MPa}$. Such results suggest a limit of tolerance between the osmotic potentials of -0.49 and $-1.03 \mathrm{MPa}$. Senna macranthera seedlings were more sensitive to osmotic stress, reducing shoot and radicle elongation when levels of external water stress were increased. Senna multijuga and $M$. bimucronata increased the radicle lenght at $\Psi=-0.15 \mathrm{MPa}$. Reduction of fresh weight was observed for all levels of osmotic stress in all species.

Key words: water stress, PEG 6000, germination, Leguminosae, seedlings.

\section{Introdução}

O período de germinação e crescimento inicial de plântulas é de extrema importância para a sobrevivência das espécies. O processo de germinação compreende três fases: (1) embebição, (2) pausa, durante a qual ocorrem ativações metabólicas e (3) início do crescimento com emergência da radícula através da testa da semente (Black \& El Hadi 1992).

Estudos têm sido realizados descrevendo os efeitos do estresse hídrico na germinação de sementes e crescimento de plântulas (Hadas 1976; McIntyre 1987; Voetberg \& Sharp 1991; Materechera et al. 1992). A deficiência hídrica é o fator limitante de maior significância na sobrevivência e crescimento inicial de plântulas (Blake 1983). O estresse hídrico pode afetar a germinação provocando um atraso do início do processo ou uma diminuição na germinabilidade final (Palit \& Bhattacharyya 1981; Hardegree \& Emmerich 1990).

Uma das técnicas mais utilizadas para avaliar o efeito consiste em simular condições de estresse, utilizando soluções com diferentes potenciais osmóticos $(\psi)$ (Taylor et al. 1982). O polietileno glicol (PEG) é utilizado na simulação do estresse hídrico por ser inerte e não causar danos metabólicos nas sementes (Cordero \& Di Stéfano 1991).

Senna macranthera DC. e S. multijuga Rich são leguminosas arbóreas nativas, muito utilizadas como ornamentais para arborização urbana e rural, devido à beleza de suas flores (Rizzini \& Mors 1976). Senna multijuga é uma das poucas árvores que resiste à zona de tensão ecológica, mantendo-se em arredores de áreas de mineração de carvão. Mimosa bimucronata (DC.) OK (maricá) é uma leguminosa de hábito arbustivo característica do Rio Grande do Sul e de acentuado valor ecológico (Ferreira 1976), sendo uma das espécies apropriadas para reflorestamento em áreas degradadas (Reitz et al. 1988).

O objetivo deste trabalho foi avaliar o efeito de diferentes potenciais osmóticos simulados com PEG 6000 na germinação e crescimento inicial de plântulas das três espécies de leguminosas anteriormente citadas.

\section{Material e métodos}

Frutos de Senna multijuga (Rich.) Irwin \& Barneby; Senna macranthera (Colladon) Irwin e Barneby \& Mimosa bimucronata (DC.) Kuntze foram coletadas nas cercanias de Porto Alegre. Em laboratório as sementes foram extraídas dos legu- 
mes e acondicionadas em frascos vedados até o uso, sendo que esta estocagem não foi superior a dois meses.

Para os testes de germinação as sementes foram desinfestadas com solução de hipoclorito de sódio $2 \%$ por 10 minutos. A seguir, foram lavadas em água destilada estéril, escarificadas mecanicamente e divididas em:

a- sementes para testar o efeito do PEG 6000 na germinação;

b- sementes para testar o efeito do PEG 6000 no crescimento das plântulas.

Todas as sementes foram colocadas em placas de Petri ( $9,5 \mathrm{~cm}$ de diâmetro) com 2 folhas de papel filtro umedecido com água destilada ou solução de PEG 6000.

a- Efeito do PEG 6000 na germinação - As sementes de cada espécie foram distribuídas em 4 tratamentos com 3 repetições de 20 sementes por placa.

Diferentes potenciais osmóticos foram simulados com uso de PEG 6000 nas seguintes concentrações: controle (água destilada); 100g/l (-0,15MPa); 200g/l $(-0,49 \mathrm{MPa})$ e $300 \mathrm{~g} / \mathrm{l}(-1,03 \mathrm{MPa})$. Os potenciais osmóticos das soluções foram calculados segundo a equação proposta por Michel \& Kaufmann (1973).

As placas de Petri com as sementes foram mantidas em câmara de germinação com temperatura de $25 \pm 1^{\circ} \mathrm{C}$ e fotoperíodo de $12 \mathrm{~h}\left(1500 \mathrm{lux}=4 \mathrm{~W} \cdot \mathrm{m}^{-2}\right)$.

O critério de germinação adotado foi emergência da radícula (Labouriau 1983). $\mathrm{O}$ número de sementes germinadas foi avaliado diariamente durante 10 dias e o potencial germinativo foi medido pela percentagem final de germinação.

As sementes de Mimosa bimucronata atingem o máximo de germinação em 48 horas, quando são escarificadas mecanicamente (Ferreira 1976). Por esse motivo, a observação foi realizada de $12 \mathrm{em} 12$ horas durante 10 dias.

b- Efeito do PEG 6000 no crescimento das plântulas — Cerca de 150 sementes de cada espécie em estudo foram colocadas para germinar em água destilada sob as condições descritas anteriormente. O tempo mínimo para obtenção de plântula foi estabelecido em 5 dias para Mimosa bimucronata e Senna multijuga e 8 dias para $S$. macranthera. Após atingido esse tempo mínimo, 90 plântulas foram tomadas aleatoriamente e nelas realizadas as medidas iniciais de peso seco e comprimento da parte aérea e da radícula. As plântulas foram distribuídas em placas gerbox com 6 repetições de 5 plântulas por placa para cada espécie estudada. Foram testados os mesmos tratamentos de solução de PEG 6000 descritos no item anterior. As placas foram distribuídas ao acaso dentro da câmara de cultivo sob as mesmas condições descritas para o teste de germinação.

A aplicação das soluções de PEG 6000 foi gradual até atingir a concentração desejada, ou seja, as plântulas permaneceram 2 horas em cada concentração intermediária. No oitavo dia o experimento foi encerrado, obtendo-se os valores finais de comprimento de radícula e parte aérea e peso das plântulas.

Análise estatística dos dados - Os dados foram submetidos ao teste de homogeneidade de variâncias de Bartlett, seguido pelo teste de Mann-Whithney para comparar os diversos tratamentos $(\alpha=0.05)$ (Sokal \& Rohlf 1981). 


\section{Resultados e discussão}

a- Efeito do PEG 6000 na germinação - De acordo com os resultados obtidos é possível constatar que não houve diferença significativa no percentual final de germinação de sementes de Senna macranthera entre os potenciais osmóticos de 0 a $-0,49 \mathrm{MPa}$ (Fig. 1A). EmS. multijuga e Mimosa bimucronata o potencial de -0,49MPa foi responsável por um decréscimo significativo na germinabilidade final ( 20 e 63,3\% respectivamente) (Fig. 1B e C). Independentemente da espécie testada, as sementes submetidas ao potencial osmótico mais negativo $(\Psi=-1,03 \mathrm{MPa})$ não ger-minaram, sugerindo existir um limite de tolerância ao déficit hídrico situado entre os potenciais de -0,49 e-1,03MPa. Segundo Hadas \& Russo (1974), a taxa de germinação decresce com a diminuição do potencial hídrico externo e para cada espécie há um valor crítico de $\Psi$, abaixo do qual a germinação não ocorrerá.
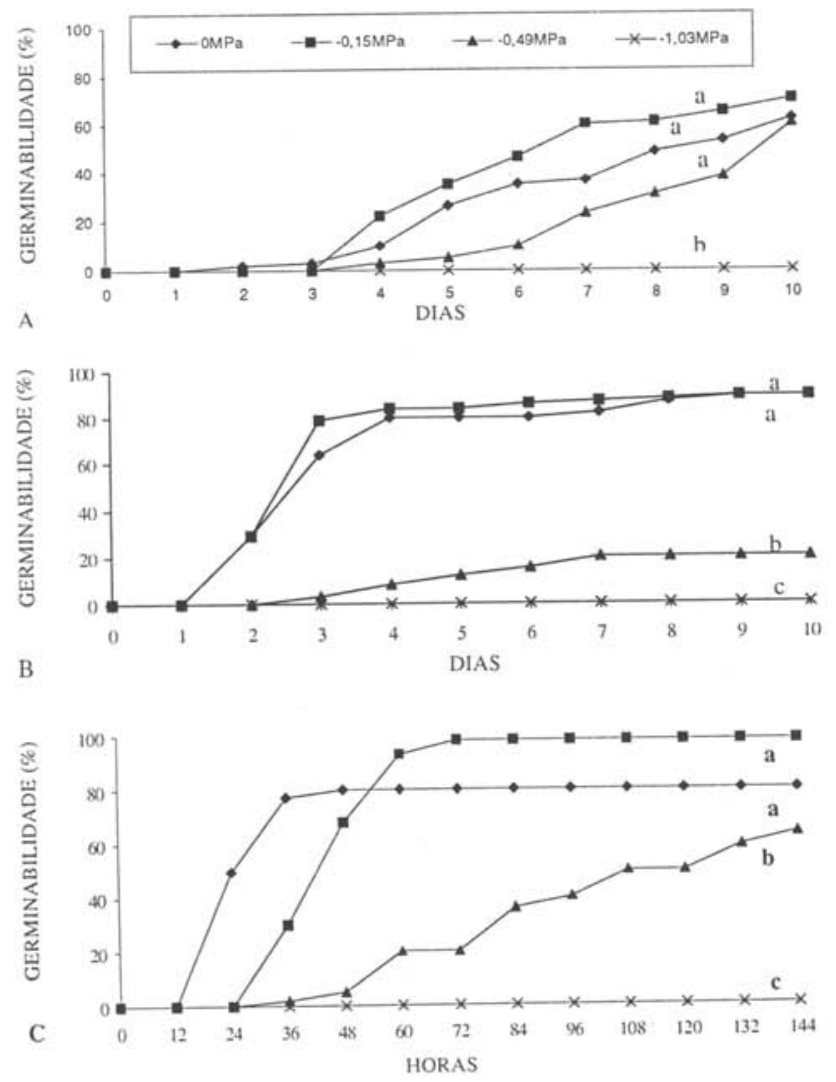

Figura 1. Efeito de diferentes potenciais osmóticos sobre o percentual acumulado de germinação de sementes de Senna macranthera (A). S. multijuga (B) e Mimosa bimucronata (C). Curvas seguidas de mesma letra não diferem entre si pelo Teste de Mann-Whithney $(\alpha=0,05)$. 
A ocorrência de danos metabólicos nas sementes tratadas com solução de $\Psi=$ -1,03MPa foi descartada em Mimosa bimucronata, pois as sementes desta espécie apresentaram germinabilidade de $90 \%$ após serem transferidas para água destilada (Borghetti comunicação pessoal). Sementes de Tecoma stans (Bignoniaceae) prétratadas com potenciais osmóticos tão negativos quanto $-1,0$ a $-1,5 \mathrm{MPa}$, ao serem transferidas para água destilada, apresentaram germinabilidade mais elevada que o controle, indicando a inexistência de danos metabólicos permanentes causados pelas soluções de PEG 6000 (Cordero \& Di Stéfano 1991). Esses resultados reforçam a confiabilidade do polietileno glicol como indutor inerte de estresse hídrico (Hardegree \& Emmerich 1990).

Com base nos resultados da figura 1A, as sementes de Senna macranthera submetidas a $\Psi=-0,15$ ou $-0,49 \mathrm{MPa}$ germinaram com $48 \mathrm{~h}$ de atraso em relação ao controle. Foi constatado um comportamento semelhante em Mimosa bimucronata e Senna multijuga, sendo o período de retardo da germinação de 12 e 24 horas, respectivamente (Fig. 1B e C). Resultados semelhantes foram obtidos por Hadas (1976) em germinação de Cicer arietinum L. (Leguminosae). O autor sugere que o potencial hídrico externo esteja relacionado com a cinética das reações enzimáticas. $\mathrm{O}$ atraso na protusão da radícula pode ter sido provocado por um retardo na velocidade das reações enzimáticas nas regiões dos meristemas, prolongando o período de pausa.

As sementes submetidas a potenciais osmóticos mais negativos apresentam um atraso no início da germinação, pois requerem mais tempo para ajustar o potencial osmótico interno de acordo com o meio externo. Esse ajuste é realizado através dos polímeros de reserva, aumentando as quantidades dos açúcares osmoticamente ativos (Taylorson 1986). O acúmulo de solutos em resposta ao estresse hídrico parece ser um dos mais efetivos mecanismos de tolerância à seca em plantas (Tan et al. 1992).

A simulação de potenciais negativos como $\Psi-0,15 \mathrm{MPa}$ mostrou uma tendência de promoção da germinação (Fig. 1A,B e C). Esse fenômeno pode ser explicado pela propriedade que o PEG 6000 apresenta de evitar a rápida absorção de água impedindo a injúria das membranas celulares e, por conseqüência, a perda de íons (Taylorson 1986). Os valores de germinabilidade obtidos $\operatorname{com} \Psi=-0,49 \mathrm{MPa}$ para $M$. bimucronata e Senna macranthera demonstram haver uma maior resistência ao estresse hídrico destas espécies em relação a $S$. multijuga.

b- Efeito do PEG 6000 no crescimento das plântulas — Os valores médios iniciais de comprimento e peso obtidos de plântulas das 3 espécies estudadas estão apresentados na tabela 1 .

Tabela 1. Plântulas de Senna macranthera com 8 dias e de $S$. multijuga e de Mimosa bimucronata com 5 dias. Média e desvio padrão das medidas iniciais de peso, comprimento da radícula e da parte aérea obtidas de 90 plântulas.

\begin{tabular}{lrll}
\hline Parâmetros & S.macranthera & S.multijuga & M.bimucronata \\
\hline Peso fresco $(\mathrm{mg})$ & $180,1 \pm 26,7$ & $48,1 \pm 9,8$ & $39,7 \pm 9,7$ \\
Radícula $(\mathrm{mm})$ & $45,9 \pm 17,3$ & $11,1 \pm 4,1$ & $20,7 \pm 10,7$ \\
Parte aérea $(\mathrm{mm})$ & $30,2 \pm 5,3$ & $24,5 \pm 5,6$ & $21,0 \pm 5,8$ \\
\hline
\end{tabular}


Nas três espécies houve decréscimo do crescimento da parte aérea com redução dos potenciais osmóticos (Fig. 2). No $\Psi=-1,03 \mathrm{MPa}$, Senna multijuga apresentou o maior crescimento de parte aérea. Os valores negativos observados nas figuras $2,3 \mathrm{e}$ 4 correspondem a uma grande perda de turgor, ocasionando a redução drástica de todos os parâmetros observados.

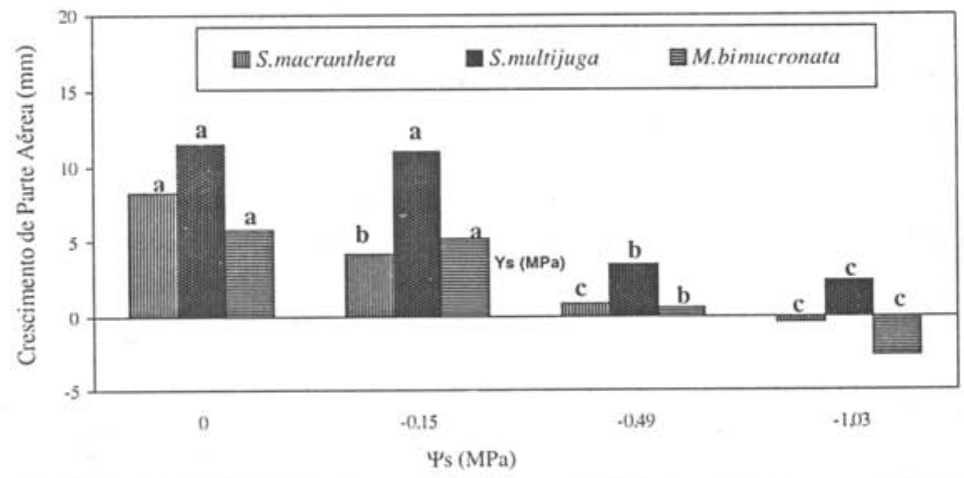

Figura 2. Efeito do PEG 6000 sobre o crescimento inicial de parte aérea em plântulas de Senna macranthera, S. multijuga e Mimosa bimucronata. Os valores apresentados são médias das diferenças entre as medidas finais e iniciais de comprimento da parte aérea. Letras diferentes indicam diferença significativa dentro de cada espécie $(\alpha=0,05)$.

As plântulas de Senna macranthera responderam com uma redução do crescimento radicular à medida que os potenciais osmóticos tornaram-se mais negativos. Nas demais espécies, o comprimento médio da radícula apresentou valores maiores no $\Psi=$ $-0,15 \mathrm{MPa}$, não atingindo valores médios negativos no potencial -1,03MPa (Fig. 3).

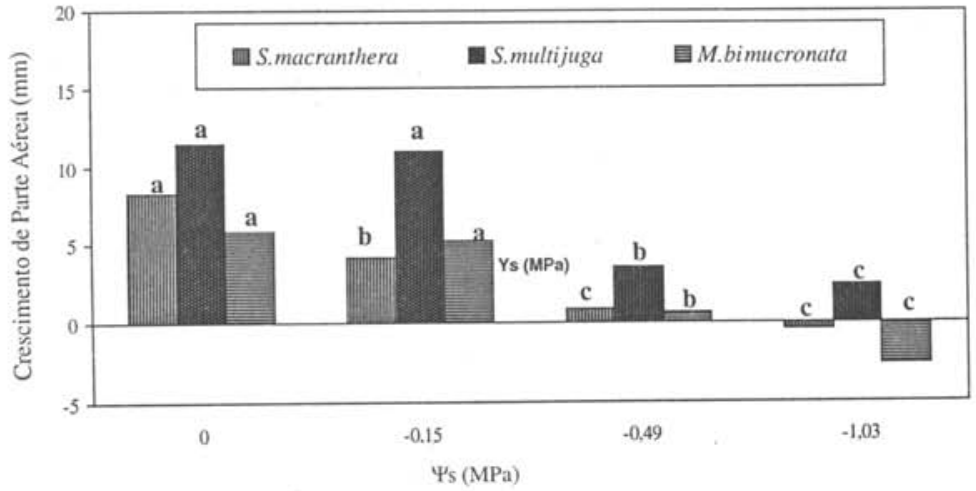

Figura 3. Efeito do PEG 6000 sob o crescimento inicial de radícula em plântulas de Senna macranthera, $S$. multijuga e Mimosa bimucronata. Os valores apresentados são médias das diferenças entre as medidas finais e iniciais do comprimento da radícula. Letras diferentes indicam diferença significativa dentro de cada espécie $(\alpha=0,05)$. 
Plântulas de Mimosa bimucronata apresentaram maior crescimento da radícula quando comparado ao crescimento da parte aérea (Fig. 2 e 3). Spollen \& Sharp (1991) relataram que raízes de milho podem crescer em níveis de potenciais que inibem o crescimento de parte aérea. Isso parece ser vantajoso para a sobrevivência da plântula quando o suprimento de água é limitado. Os autores sugerem ainda que a plântula sob estresse hídrico aumenta a concentração de $\mathrm{ABA}$ endógeno, e esse atuaria tanto mantendo o crescimento da raiz, quanto inibindo o crescimento da parte aérea. Foi observado um comportamento inverso em Senna multijuga, a qual apresentou crescimento de parte aérea maior que o radicular. Segundo Spyropoulos (1986), o estresse hídrico pode causar redução no crescimento de plântulas por restringir o afrouxamento da parede celular ou por reduzir a entrada de solutos para o embrião. Sob condições de estresse hídrico, o autor observou uma baixa atividade de enzimas na degradação da sacarose, resultando em um decréscimo no crescimento do embrião.

A redução do peso de plântulas de Senna macranthera foi observada já no $\Psi=$ $-0,15 \mathrm{MPa}$, sendo mais acentuada nos potenciais mais negativos. Senna multijuga e Mimosa bimucronata apresentaram uma significativa redução de peso a partir do $\Psi=$ -0,49MPa (Fig. 4). Roundy et al. (1989) relataram um decréscimo de peso de parte aérea e raiz de arroz para potenciais entre $-0,5$ e -1,0MPa.

Com base nos resultados obtidos, sugere-se, para estudos futuros, que os intervalos entre as concentrações estabelecidas sejam menores, visando determinar o limite de cada espécie.

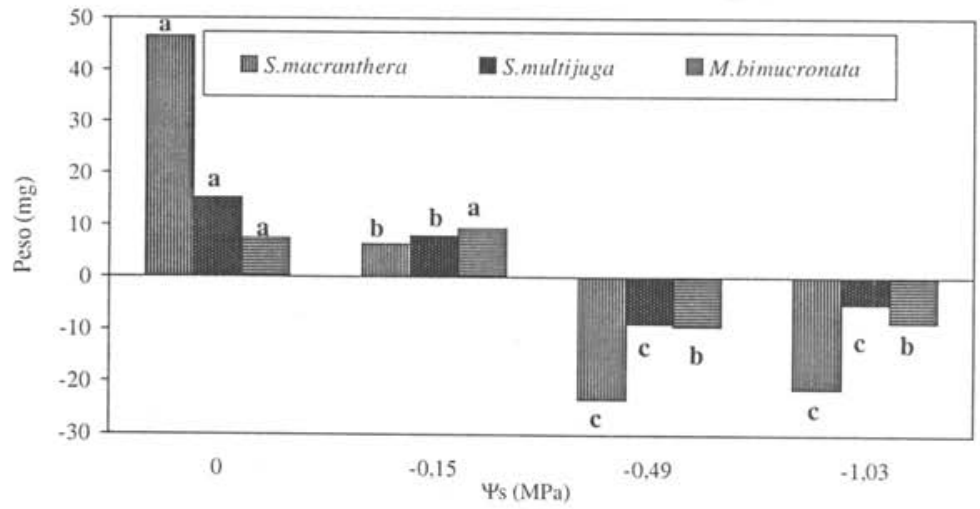

Figura 4. Efeito do PEG 6000 sobre o peso de plântulas de Senna macranthera, S. multijuga e Mimosa bimucronata. Os valores apresentados são médias das diferenças entre as medidas finais e iniciais do peso. Letras diferentes indicam diferença significativa dentro de cada espécie $(\alpha=0,05)$.

Os resultados indicam que Mimosa bimucronata foi a espécie mais tolerante ao estresse hídrico quando se considera germinação e crescimento inicial de plântulas. Essa tolerância fica evidenciada pela capacidade de crescimento radicular, mesmo em 
potenciais mais negativos, danosos às demais espécies estudadas. Esta constatação é coerente com a capacidade da espécie de se estabelecer em regiões degradadas e com seu caráter de ser planta pioneira. Seria interessante associar estudos anatômicos e bioquímicos ao processo de estresse hídrico para investigar quais os mecanismos utilizados pela Mimosa bimucronata para tolerar baixos potenciais osmóticos.

\section{Agradecimentos}

À Dr. ${ }^{a}$ Lucia Dillenburg pela leitura crítica do manuscrito.

\section{Referências bibliográficas}

Black, A.R. \& El Hadi, F.M. 1992. Presouring treatments of Acacia senegal seed: germination and growth. Trop. Agric. 69: 15-20.

Blake, T.J. 1993. Transplanting shock in white spruce; effect of cold storage and root pruning on water relations and stomatal conditioning. Physiol. Plant. 57: 210-216.

Cordero, R.A.S. \& Di Stéfano, J.F.G. 1991. Efecto del estrés osmótico sobre la germinación de semillas de Tecoma stans (Bignoniaceae). Rev. Biol. Trop. 39: 107-110.

Ferreira, A.G., 1976, Germinação de sementes de Mimosa bimucronata (DC) OK (maricá) - Efeito da escarificação e do pH. Ciência e Cultura 28: 1200-1204.

Hadas, A. 1976. Water uptake \& germination of leguminous seeds under changing external water potential in osmotic solutions. J. Exp. Bot. 27: 480-489.

Hadas, A. \& Russo, D. 1974. Water uptake by seeds as affected by water stress, capillary condutivity and seed-soil water contact. I- Experimental study. Agron. J. 66: 643-647.

Hardegree, S.P. \& Emmerich, W.E. 1990. Effect of polyethylene glycol exclusion on the water potential of solution saturated filter paper. Plant. Physiol. 92: 462-466.

Labouriau, L.F.G. 1983. A germinação de sementes. Ed. Sec. Org. dos Estados Unidos, Washington, D.C. $174 \mathrm{p}$.

Materechera, S.A.; Dexter, A.R.; Alston, A.M. \& Kirby, J.M. 1992. Growth of seedling roots in response to external osmotic stress by polyethylene glycol 20,000. Plant and Soil 143: 85-91.

Mcintyre, G.I. 1987. The role of water in the regulation of plant development. Can. J. Bot. 65: 1287-1298.

Michel, B.E. \& Kaufmann, M.R. 1973. The osmotic potential of polyethylene glycol 6000. Plant Physiol. 51: $914-916$.

Palit, P. \& Bhattacharyya, A.C., 1981, Germination and water uptake of jute seeds under water stress. Ind. J. Exp. Biol. 19: 848-852.

Roundy, B.A.; Young, J.A. \& Evans, R.A. 1989 Seedling growth of three great wild rye colletions at reduced osmotic potential. Agric. Ecos, and Envir. 25: 245-251.

Reitz, R.; Klein, R.M. \& Reis, A. 1988. Projeto Madeira do Rio Grande do Sul. CORAG, Porto Alegre. 525 p.

Rizzini, C.T. \& Mors, W.B. 1976. Botânica Econômica Brasileira. EDUSP, São Paulo. 207 p.

Sokal, R.R. \& Rohlf, F.J. 1981. Biometry. FREEMAN W.H. \& Co.,San Francisco. 859 p.

Spollen, W.G. \& Sharp, R.E. 1991. Spatial distribution of turgor and root growth of low water potential. Plant Physiol. 96: 438-443.

Spyropoulos, C.G. 1986. Osmoregulation, growth and sucrose accumulation in germinated Trigonella foenum-graecum (fenugreek) seed treated with polyethylene glycol. Physiol. Plant. 68: 129-135.

Tan, W.; Blake, T.J. \& Boyle, T.J.B. 1992. Drought tolerance in faster and slower growing spruce (Picea mariana) progenies: II- Osmotic adjustment and changes of soluble carbohydrates and amino acids under osmotic stress. Physiol. Plant. 84: 645-651.

Taylor, A.G.; Motes, J.E. \& Kirkham, M.B. 1982. Germination and seedling growth characteristics of 
three tomato species affected by water deficits. J. Am. Soc. Hort. Sci. 107: 282-285.

Taylorson, R.V. 1986. Water stress - Induced germination of giant foxtail (Setaria faberi) seeds. Weed Sci. 34: 871-875.

Voetberg, G,S. \& Sharp, R.E. 1991. Growth of maize primary root at low water potentials. Plant Ply:siol. 96: 1125-1130. 